\title{
Support from Biomedical Expert Improves Nursing Care - Opinion Based on Experience
}

\author{
Sarmad Muhammad Soomar* \\ School of Nursing \& Midwifery, Aga Khan University, Karachi Pakistan

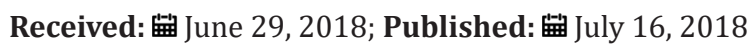

*Corresponding author: Sarmad Muhammad Soomar, Rho Delta Chapter, Sigma (office first floor) - Aga Khan University, School of Nursing \& Midwifery, Karachi Pakistan

Keywords: Biomedical; Neonatal; Auxiliary; Syringe; Ventilators; Oxygenation; Nutrition; Medication; Cardiac Monitor; Pediatric

\section{Opinion}

Working around a hospital as ac nurse makes you work with equipment and machines always. Whatever we learnt in the nursing school was theoretically based on the understanding of what is a disease, what are procedures and what related care should be given to the clients. But the scenario changed when we kept our feet in the clinical areas during electives, independent clinical or mainly after graduation. What I personally realized that a nurse is more a technician at the ward setting in practical aspects along with a compassionate care giver.Involving in maintenance of feeds with pumps, handling ventilators, using equipment for cardiac monitoring, electronic screens for vital signs, technical pumps to start infusion and many more examples are all related to the daily routine work of a tertiary care hospital nurse. All major care that involves nutrition, medication, monitoring, oxygenation etc., is depended on machines and equipment.Realizing that this kind of care process in which all nurses are practicingis tough as more of the schooling work was based on written exams or understanding text about major tasks.

That merely not involved working on machines or their simulators to practice before entering into real patient setting. The most funny thing I realized from my time is injecting an intramuscular to a filthy soft foam or sponge that already had many areas torn, understanding about an intensive care ventilator through a research study article publish somewhere in Europe, learning how to operate a infusion pump with a group of fifty students in a small room and sitting in a last row, while watching a blur video and knowing about a cardiac balloon and its management through a discussion, even you haven't see it still. What insights these moments gave me as a student that without touching those things in actual how my fear of work will reduce, and how to make the learning possible to attain a maximum level of handling these timely changing equipment and technical stuff. As a nurse you remember the dose but don't know how to set the syringe in pump or you are expert in recalling basic vital signs of every age but don't remember the alarm setting codes than its troublesome in terms of working and standing in the area of patient care.

For this purpose and to solve this issue a trained biomedical personal for wards and hospitals is necessary, who is an expert of equipment with a degree. In some context of Pakistan trained technicians for a specific time, a month or two are designated to do these tasks which usually don't work. A specific biomedical person can do this only with his expertise, not any other and nurses should not be expected fully to know all these machinesbut they should be monitored and evaluated timely by biomedical team to see how they have improved the practices regarding technical items in their patient care. Are they able to notify error, do they understand default changes or they are competent enough to manage and settle the parameters of specific machines, pumps or equipment. Registered nurses with a diploma or a degree can be specifically trained by their working hospital once they complete their educational requirements. These trainings and evaluation should be taken by specific experts of biomedical, who mainly deal the handling of this equipment. Culture of creating technicians in specific clinical setting helps nurses temporarily but doesn't enhance their learning so they can handle in their absence.

Also technicians in many contexts are nursing aid or other auxiliary staff who has additionally given these learning to support nurses but not experts who can periodically train nurses and monitor their understanding.This opinion generated from my experience with my study colleagues at a tertiary hospital in Pakistan during our electives. Wenoticed that the neonatal nurses have less understanding of parameters and alarm setting in a cardiac monitor. Many nurses had managed their monitors with only pediatric or adult setting or alarms, where as their monitors have specific setting of neonates. Directly attempting to share with them about these kinds of mistakes was not a good option instead we talked to their head nurse and asked him to arrange a biomedical expert. Taking this step really supported my team's 
opinion that a biomedical expert is a good support to nurses in terms of learning equipment and machines instead of any other technician or any other person. Because not only they shared the text of maintaining and handling these technical itemsbut gave individual demonstration and took re- demonstration.

This experience gave us two opportunities, number one was of self-learning about equipment in neonatal setup so that we can help other nurses during our electives and second was assessing and evaluating working nurses on the basis of practical questions like show us how you will check this particular parameter or set this kind of alarm etc.On the basis of assessing them, we were able to share them that something is lacking in their practices because they we not aware with actual technical setting of the cardiac monitors and other equipment used in their settings. With record of assessments and checklists filled, we were able to put our opinion in front that periodical trainings and evaluation is necessary with support of a biomedical expert so that nurses can improve care and quality standards of care and patient satisfaction.

We were also fortunate that the leadership was also keenly interested in keeping this opinion at fore front and work in their setting related to theneed of biomedical support for nurses. This also provided us the opportunity to conduct the initial training of nurses with our learnings taken from biomedical experts and we did the same in presence of the leadership and biomedical experts to take an account of required learning for the nurses.
ISSN: 2574-1241

DOI: 10.26717/BJSTR.2018.06.001416

Sarmad Muhammad Soomar. Biomed J Sci \& Tech Res

CC This work is licensed under Creative

Submission Link: https://biomedres.us/submit-manuscript.php

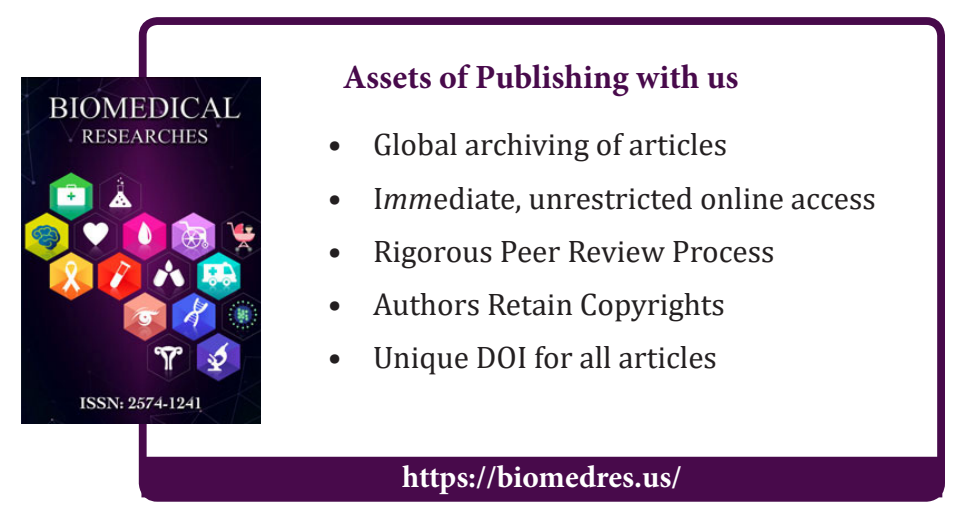

